\title{
Potential beneficial effects of oral administration of isoflavones in patients with chronic mountain sickness
}

\author{
JIANHUA CUI ${ }^{1}$, LIANG GAO ${ }^{1}$, HAIJUN YANG ${ }^{1}$, FULING WANG ${ }^{1}$, CHUNHUA JIANG $^{2,3,4}$ and YUQI GAO $^{2,3,4}$ \\ ${ }^{1}$ Research Center of PLA for Prevention and Treatment of High Mountain Sickness, The 18th Hospital of PLA, \\ Yecheng, Xinjiang 844900; ${ }^{2}$ Department of Pathophysiology and High Altitude Physiology, \\ College of High Altitude Military Medicine, Third Military Medical University; ${ }^{3}$ Key Laboratory of High Altitude Medicine, \\ Ministry of Education; ${ }^{4}$ Key Laboratory of High Altitude Medicine, PLA, Chongqing 400038, P.R. China
}

Received July 7, 2013; Accepted October 30, 2013

DOI: $10.3892 /$ etm.2013.1388

\begin{abstract}
Soy isoflavones (Ifs), which are natural phytoestrogens, have beneficial effects in cardiovascular disease. We have previously shown that genistein, the most active component of Ifs, inhibits pulmonary vascular structural remodeling and right ventricular hypertrophy induced by chronic hypoxia in male Wistar rats. This study aimed to evaluate the effects of Ifs on right ventricular and pulmonary hemodynamics in individuals with chronic mountain sickness (CMS). Twenty-eight male patients living on the Qinghai-Tibetan plateau $(5,200 \mathrm{~m})$ who were suffering from CMS were treated orally with Ifs (20 mg, twice daily) for 45 days. Physiological and plasma biochemical indices, hematology and echocardiography were investigated. It was observed that 45 days of treatment with Ifs significantly increased blood oxygen saturation and markedly decreased the CMS score and heart rate (all $\mathrm{P}<0.05)$ of the subjects. Following treatment with Ifs, hematocrit $(\mathrm{P}<0.05)$, hemoglobin concentration $(\mathrm{P}<0.01)$ and plasma levels of malondialdehyde $(\mathrm{P}<0.05)$ were significantly decreased, while plasma levels of nitric oxide $(\mathrm{P}<0.01)$ and the plasma activity of nitric oxide synthase $(\mathrm{P}<0.01)$ and superoxide
\end{abstract}

Correspondence to: Professor Yuqi Gao or Dr Chunhua Jiang, Department of Pathophysiology and High Altitude Physiology, College of High Altitude Military Medicine, Third Military Medical University, No. 30 Gao Tan Yan Zheng Street, Chongqing 400038, P.R. China

E-mail: gaoy66@gmail.com

E-mail: 38068549@qq.com

Abbreviations: CMS, chronic mountain sickness; Acz, acetazolamide; Ifs, isoflavones; NO, nitric oxide; NOS, nitric oxide synthase; SOD, superoxide dismutase; MDA, malondialdehyde; MPA, main pulmonary artery diameter; RVEAD, right ventricular end-diastolic anteroposterior diameter; RVETD, right ventricular end-diastolic trans diameter; RVAW, right ventricular anterior wall; RVOT, right ventricular outflow tract

Key words: high altitude, chronic mountain sickness, isoflavone, erythrocytosis, pulmonary hemodynamics dismutase $(\mathrm{P}<0.01)$ were markedly increased compared with the respective values obtained prior to treatment with Ifs. The echocardiography results showed that Ifs significantly decreased the main pulmonary artery diameter $(\mathrm{P}<0.05)$, right ventricular end-diastolic anteroposterior diameter $(\mathrm{P}<0.01)$, right ventricular end-diastolic trans diameter $(\mathrm{P}<0.01)$, right ventricular anterior wall $(\mathrm{P}<0.01)$ and right ventricular outflow tract $(\mathrm{P}<0.01)$. These results indicate the potential beneficial effects of Ifs in the reduction of excessive erythrocytosis, the alleviation of oxidative damage and the amelioration of right ventricular index and pulmonary hemodynamics in CMS.

\section{Introduction}

Chronic mountain sickness (CMS) or Monge's disease, first described by Carlos Monge Medrano in Peru in 1925 (1), is characterized by excessive erythrocytosis (a hemoglobin level of $\geq 19 \mathrm{~g} / \mathrm{dl}$ in females or $\geq 21 \mathrm{~g} / \mathrm{dl}$ in males) in combination with chronic hypoxemia; in certain cases pulmonary hypertension is also apparent (2-4). Severe pulmonary hypertension may evolve to cor pulmonale, resulting in congestive heart failure. Therefore, CMS frequently leads to right cardiac failure and/or neurological disorders (3-5). The clinical signs in patients with CMS include breathlessness and/or palpitations, sleep disturbance, cyanosis, dilatation of veins, paresthesia, headache and tinnitus $(2,4)$. On the Qinghai-Tibetan plateau, the incidence of CMS is $2.43-37.5 \%(3,000-4,700 \mathrm{~m})$ and increases with elevation $(4,6)$. Furthermore, the prevalence of CMS is significantly increased in migrants compared with that in the native population of high altitudes, and the disease occurs more frequently in males than in females (7-9). CMS is a severe public health problem for the Qinghai-Tibetan plateau, which has $>60$ million people in residence (4).

Despite a number of trials with medroxyprogesterone, enalapril and almitrine, no pharmacological treatment is currently widely used to impede the progressive loss of adaptation to chronic hypoxia (10-13). Acetazolamide (Acz) is useful for reducing hematocrit and improving pulmonary circulation in CMS. However, Acz has adverse effects, including paresthesia and diuresis (14). Therefore, the only management for CMS, at present, is repetitive bloodletting or the displacement of dwellers to lower altitudes. The latter option has an important 
negative social and economic impact on the development of high-altitude regions.

Excessive erythropoiesis in CMS is a process that is hormonally regulated $(15,16)$. High serum testosterone levels are correlated with excessive erythrocytosis in males at high altitude, and female sex hormones suppress polycythemic and cardiopulmonary responses in vivo during chronic hypoxic exposure $(16,17)$. It is generally accepted that an excessive number of red blood cells in the vessels is likely to increase blood viscosity, thereby inducing pulmonary hypertension and even cardiac failure. However, the underlying mechanism of pulmonary hypertension in CMS is only partially understood. Data from Bailey et al (18) indicated that increased pulmonary arterial systolic pressure at high altitude was correlated with a reduction in pulmonary nitric oxide (NO) bioavailability (18). Therefore, the inhibition of erythropoiesis and the increase of circulating NO levels are likely to alleviate the clinical signs of patients with CMS.

Soy isoflavones (Ifs) are the most studied and most potent phytoestrogens, and are predominantly present in soy-based foods (19). Genistein and daidzein, the two most abundant Ifs (19), have chemical structures similar to that of estradiol (E2) and exert estrogen-like effects (20), which are able to inhibit excessive erythropoiesis. Experimental studies have shown the beneficial effects of Ifs on the production of NO in endothelial cells, which include inhibition of the proliferation of vascular smooth muscle and reduction of the tissue damage caused by reactive oxygen species (ROS) $(21,22)$. Animal studies have also indicated that genistein and daidzein, through estrogen receptor (ER)-independent mechanisms, restore endothelial cell function by increasing $\mathrm{NO}$ production and the protection of $\mathrm{NO}$ from $\mathrm{O}_{2}{ }^{-}$-driven inactivation (23). In addition, our previous studies showed that treatment with genistein inhibited pulmonary vascular structural remodeling and right ventricular hypertrophy induced by chronic hypoxia (5,000 $\mathrm{m}, 21$ days) in male Wistar rats without adverse effects, such as feminizing and oncogenic effects (24-26).

Therefore, in the present study it was hypothesized that Ifs may reduce excessive erythropoiesis and ameliorate right ventricular index and pulmonary hemodynamics in CMS. The study aimed to observe the effects of Ifs in patients with CMS and to evaluate the treatment of CMS.

\section{Materials and methods}

Subjects and procedure. Twenty-eight male patients suffering from CMS, who were living on the Qinghai-Tibetan plateau $(5,200 \mathrm{~m})$, gave their informed written consent for this study, which was approved by the Ethics Committee of the Third Military Medical University (Chongqing, China). The Ethics Committee of the Third Military Medical University refused to permit a study design where no treatment was administered to patients who had been diagnosed with CMS with malaise. Therefore, placebo treatment was not given to the patients with CMS. This study was only designed to observe the effects of Ifs on patients with CMS by comparing various parameters prior to and following treatment. All subjects, who were born and previously lived in a low-altitude area, were migrants of the Qinghai-Tibetan plateau $(5,200 \mathrm{~m})$, had been residing at the plateau $>10$ months and did not travel to lower altitudes $(<5,000 \mathrm{~m})$. All patients had a hemoglobin concentration $\geq 21 \mathrm{~g} / \mathrm{dl}$ and were working at the same labor intensity. A complete clinical evaluation, including an evaluation of ventilatory function and cardiac ultrasonography, was performed prior to inclusion in the study to exclude subjects with cardiac or pulmonary diseases. The age, weight and height of the subjects were $20.86 \pm 1.53$ years, $66.36 \pm 6.59 \mathrm{~kg}$ and $1.76 \pm 0.35 \mathrm{~m}$, respectively. The CMS clinical score was calculated according to a consensus statement on chronic high-altitude diseases (2) and was based on the following symptoms: breathlessness and/or palpitation, sleep disturbance, cyanosis, dilatation of veins, paresthesia, headache and tinnitus. Finger blood oxygen saturation $\left(\mathrm{SaO}_{2}\right)$ and heart rate (HR) were measured using a TuffSat handheld oximeter (GE Healthcare, Fairfield, CT, USA) following a 15-min rest in the sitting position. Subjects were treated orally with Ifs capsules (containing $20 \mathrm{mg}$ mixed Ifs, twice daily; Department of Traditional Chinese Medicine Preparation, Xinqiao Hospital, Third Military Medical University, Chongqing, China) for 45 days. All measurements were obtained at low altitude on two occasions, prior to and following treatment with Ifs.

Hematological status. Anticoagulated peripheral venous blood samples were obtained from subjects at 5,200 $\mathrm{m}$ in the sitting position, immediately subsequent to the subjects waking up in the morning. Hemoglobin concentration was obtained using cyanide measurements and a duplication was performed for each sample. The measurements for hematocrit were obtained using Symex pocH-80i automatic blood cell analyzer (Sysmex, Kobe, Japan). Following centrifugation at $1,000 \mathrm{x} \mathrm{g}$ for $10 \mathrm{~min}$, the plasma was collected and stored at $-20^{\circ} \mathrm{C}$. The levels of plasma NO, nitric oxide synthase (NOS), superoxide dismutase (SOD) and malondialdehyde (MDA) were measured using corresponding chemical colorimetry reagent kits (Nanjing Jiancheng Bioengineering Institute, Nanjing, China), in accordance with the manufacturer's instructions.

Echocardiographic measurements. Echocardiography was performed with a 2.5-MHz transducer (Logiq Book XP, GE Healthcare) to evaluate the main pulmonary artery diameter (MPA), right ventricular end-diastolic anteroposterior diameter (RVEAD), right ventricular end-diastolic trans diameter (RVETD), right ventricular anterior wall (RVAW) and right ventricular outflow tract (RVOT) by a single experienced echocardiographer, as previously described (27).

Statistical analysis. Data are expressed as the mean \pm standard deviation. The analyses were performed using a Student's t-test. $\mathrm{P}<0.05$ was considered to indicate a statistically significant difference.

\section{Results}

Effects of treatment with Ifs on physiological outcomes. Following 45 days of treatment with Ifs, $\mathrm{SaO}_{2}$ was significantly increased by $2.04 \%(\mathrm{P}<0.05)$, the CMS score was decreased by 1.07 points $(\mathrm{P}<0.01)$ and $\mathrm{HR}$ was decreased by 4.17 beats $/ \mathrm{min}(\mathrm{P}<0.05)$ compared with the values prior to treatment (Table I). These changes clearly indicated an 
Table I. Effects of treatment with isoflavones on the physiological parameters of patients with CMS (n=28).

\begin{tabular}{lccr}
\hline Time-point & CMS score (points) & Heart rate (beats/min) & SaO $(\%)$ \\
\hline Prior to treatment & $4.68 \pm 1.47$ & $92.46 \pm 10.97$ & $81.07 \pm 3.39$ \\
Following treatment & $3.61 \pm 2.18$ & $88.29 \pm 10.46$ & $83.11 \pm 3.97$ \\
P-value & 0.007 & 0.042 & 0.028
\end{tabular}

Values represent the mean \pm standard deviation. P-value, comparison between values prior to and following treatment with isoflavones. CMS, chronic mountain sickness; $\mathrm{SaO}_{2}$, blood arterialized oxygen saturation.

Table II. Effects of treatment with isoflavones on the hematological outcomes of patients with CMS (n=28).

\begin{tabular}{|c|c|c|c|c|c|c|}
\hline Time-point & Hematocrit & $\begin{array}{l}\text { Hemoglobin } \\
(\mathrm{g} / \mathrm{dl})\end{array}$ & $\begin{array}{c}\text { MDA } \\
(\mathrm{nmol} / \mathrm{ml})\end{array}$ & $\begin{array}{c}\text { SOD } \\
(\mathrm{U} / \mathrm{ml})\end{array}$ & $\begin{array}{c}\text { NOS } \\
(\mathrm{U} / \mathrm{ml})\end{array}$ & $\begin{array}{c}\mathrm{NO} \\
(\mu \mathrm{mol} / 1)\end{array}$ \\
\hline Prior to treatment & $0.71 \pm 0.08$ & $22.24 \pm 0.78$ & $4.81 \pm 2.09$ & $68.10 \pm 18.56$ & $40.43 \pm 13.61$ & $47.50 \pm 14.29$ \\
\hline Following treatment & $0.67 \pm 0.05$ & $21.18 \pm 1.05$ & $3.65 \pm 2.11$ & $78.73 \pm 14.64$ & $47.62 \pm 10.87$ & $55.20 \pm 14.10$ \\
\hline $\mathrm{P}$-value & 0.012 & $<0.001$ & 0.012 & $<0.001$ & 0.002 & 0.004 \\
\hline
\end{tabular}

Values represent the mean \pm standard deviation. P-value, comparison between values prior to and following treatment with isoflavones. CMS, chronic mountain sickness; MDA, malondialdehyde; SOD, superoxide dismutase; NOS, nitric oxide synthase; NO, nitric oxide.

Table III. Effects of treatment with isoflavones on the cardiac and pulmonary circulation of patients with CMS (n=28).

\begin{tabular}{|c|c|c|c|c|c|}
\hline Time-point & $\begin{array}{l}\text { MPA } \\
(\mathrm{mm})\end{array}$ & $\begin{array}{l}\text { RVEAD } \\
(\mathrm{mm})\end{array}$ & $\begin{array}{l}\text { RVETD } \\
(\mathrm{mm})\end{array}$ & $\begin{array}{c}\text { RVAW } \\
(\mathrm{mm})\end{array}$ & $\begin{array}{c}\text { RVOT } \\
(\mathrm{mm})\end{array}$ \\
\hline Prior to treatment & $23.38 \pm 2.70$ & $23.39 \pm 3.12$ & $30.71 \pm 3.75$ & $5.89 \pm 1.22$ & $33.89 \pm 3.59$ \\
\hline Following treatment & $22.00 \pm 2.31$ & $21.66 \pm 2.49$ & $27.71 \pm 3.38$ & $5.39 \pm 0.79$ & $31.70 \pm 3.37$ \\
\hline P-value & 0.011 & $<0.001$ & $<0.001$ & 0.010 & 0.002 \\
\hline
\end{tabular}

Values represent the mean \pm standard deviation. P-value, comparison between values prior to and following treatment with isoflavones. CMS, chronic mountain sickness; MPA, main pulmonary artery diameter; RVEAD, right ventricular end-diastolic anteroposterior diameter; RVETD, right ventricular end-diastolic trans diameter; RVAW, right ventricular anterior wall; RVOT, right ventricular outflow tract.

improvement in hypoxemia and a reduction of clinical malaise in patients with CMS.

Effects of treatment with Ifs on hematological outcomes. The number of polycythemic individuals (hemoglobin $\geq 21 \mathrm{~g} / \mathrm{dl}$ ) decreased from 28 to 17 following treatment with Ifs. In addition, treatment with Ifs significantly decreased the hematocrit by $4 \%(\mathrm{P}<0.05)$, hemoglobin concentration by $1.06 \mathrm{~g} / \mathrm{dl}(\mathrm{P}<0.01)$ and plasma levels of MDA by $1.16 \mathrm{nmol} / \mathrm{ml}(\mathrm{P}<0.05)$, and markedly increased the plasma levels of NO by $7.70 \mu \mathrm{mol} / 1$ $(\mathrm{P}<0.01)$ and the activities of NOS and SOD by 7.19 and $10.63 \mathrm{U} / \mathrm{ml}$, respectively ( $\mathrm{P}<0.01$ for each; Table II). These changes indicated that Ifs reduced the hemoglobin concentration and increased the plasma NO levels and antioxidant capacity in patients with CMS.

Effects of treatment with Ifs on the cardiac and pulmonary circulation. Table III shows the echocardiographic measure- ments of the patients with CMS. Forty-five days of treatment with Ifs significantly decreased MPA by $1.38 \mathrm{~mm}(\mathrm{P}<0.05)$, RVEAD by $1.73 \mathrm{~mm}$, RVETD by $3.00 \mathrm{~mm}$, RVAW by $0.50 \mathrm{~mm}$ and RVOT by $2.19 \mathrm{~mm}$ compared with the values prior to treatment (all $\mathrm{P}<0.01$ ). These results suggested an amelioration in the pulmonary hemodynamics and right ventricular index in patients with CMS.

\section{Discussion}

CMS is an excessive polycythemia that is common among high-altitude residents, particularly in migrants (2). According to the prevalence of CMS, millions of residents on the Qinghai-Tibetan plateau suffer from CMS. Therefore, these residents may benefit from a treatment that reduces their hemoglobin concentration and pulmonary arterial pressure.

The physiopathology of CMS has been attributed to the following sequence: hypoxemia, excessive erythropoiesis and 
pulmonary hypertension $(4,7)$. In the present study, following treatment with Ifs, the hematocrit and hemoglobin concentrations of the subjects were significantly decreased compared with the values prior to treatment. Therefore, the number of polycythemic individuals (hemoglobin $\geq 21 \mathrm{~g} / \mathrm{dl}$ ) decreased from 28 to 17 following treatment with Ifs. Moreover, 45 days of treatment with Ifs significantly increased $\mathrm{SaO}_{2}$ and decreased HR and the CMS score compared with the values prior to treatment, which indicated a reduction in hypoxemia and clinical indisposition. In addition, Ifs significantly reduced MPA, RVEAD, RVETD, RVAW and RVOT. Therefore, these results indicated improvements in hypoxia and clinical indisposition, and an amelioration of the right ventricular index and pulmonary hemodynamics in patients with CMS following treatment with Ifs. Furthermore, these results were consistent with our previous observations in rats, which showed the preventive effects of phytoestrogens on chronic hypoxic pulmonary hypertension and right ventricular hypertrophy $(25,26)$. In addition, there were no adverse effects, such as diuresis, paresthesia and heartburn, following treatment with Ifs in the present study. Data from Hamilton-Reeves et al (28) and our own laboratory showed that Ifs elicited no effects on reproductive hormones in men or male rats $(26,28)$. Ifs may have a more extensive applications in patients with CMS compared with Acz, which exhibits beneficial and adverse effects.

Furthermore, the present study demonstrated that treatment with Ifs significantly decreased plasma MDA levels and increased SOD activity, indicating an attenuation of oxidative damage in patients with CMS. This may result in the relief of malaise in patients with CMS during long-term exposure to high-altitude hypoxia. Marked increases in the plasma activity of NOS and circulating NO levels were observed following 45 days of treatment with Ifs compared with the values prior to treatment. NO has been demonstrated to be important in adaptation or acclimatization to high altitude. Scherrer et al (29) revealed that NO reduced pulmonary arterial pressure and improved oxygen saturation among patients suffering from high-altitude pulmonary edema (29). Furthermore, total NO has been demonstrated to be negatively correlated with pulmonary arterial systolic pressure and positively correlated with pulmonary blood flow and oxygen delivery at 4,200 $\mathrm{m} \mathrm{(30).} \mathrm{In} \mathrm{age} \mathrm{and} \mathrm{biomarker-adjusted}$ logistic regression, NOS was also shown to be inversely predictive of CMS (31). In the present study, the increased plasma activity of NOS and circulating NO levels, induced by 45 days of treatment with Ifs, relaxed small pulmonary arteries and reduced MPA and right ventricular ejection resistance. These observations were consistent with the results from our previous studies, in which treatment with genistein, the most active form of Ifs, inhibited pulmonary vascular structural remodeling and right ventricular hypertrophy induced by chronic hypoxia $(5,000 \mathrm{~m}, 21$ days) in male Wistar rats $(25,26)$.

In conclusion, the present study demonstrated the potential beneficial effects of Ifs in reducing excessive erythrocytosis, alleviating oxidative damage and ameliorating right ventricular index and pulmonary hemodynamics in CMS. We propose that Ifs are a potential low-cost treatment for CMS. Studies with a control group and a dose-effect analysis, and large-scale trials are required to evaluate the feasibility of the implementation of treatment for CMS in the Tibetan region.

\section{Acknowledgements}

This study was supported by the 'Eleventh Five-Year' National Technology Support Program of China (grant no. 2009BAI85B01), the National Key Basic Research Development Program of China (grant no. 2012CB518201) and the National Science Foundation of China (grant nos. 30971426 and 81101469).

\section{References}

1. Monge CC: Chronic mountain sickness. Physiol Rev 23: 166-184, 1943.

2. León-Velarde F, Maggiorini M, Reeves JT, et al: Consensus statement on chronic and subacute high altitude diseases. High Alt Med Biol 6: 147-157, 2005.

3. Penaloza D and Arias-Stella J: The heart and pulmonary circulation at high altitudes: healthy highlanders and chronic mountain sickness. Circulation 115: 1132-1146, 2007.

4. Gao Y: High Altitude Military Medicine. Chongqing Publishing House, Chongqing, 2005.

5. Wood S, Norboo T, Lilly M, Yoneda K and Eldridge M: Cardiopulmonary function in high altitude residents of Ladakh. High Alt Med Biol 4: 445-454, 2003.

6. Wu TY: Chronic mountain sickness on the Qinghai-Tibetan plateau. Chin Med J (Engl) 118: 161-168, 2005.

7. Monge CC, Arrequi A and León-Velarde F: Pathophysiology and epidemiology of chronic mountain sickness. Int J Sports Med 13 (Suppl 1): S79-S81, 1992.

8. Beall CM, Brittenham GM, Macuaga F and Barragan M: Variation in hemoglobin concentration among samples of high-altitude natives in the Andes and the Himalayas. Am J Hum Biol 2: 639-651, 1990

9. Wu T, Wang X, Wei C, et al: Hemoglobin levels in Qinghai-Tibet: different effects of gender for Tibetans vs. Han. J Appl Physiol 98: 598-604, 2005.

10. Kryger M, McCullough RE, Collins D, Scoggin CH, Weil JV and Grover RF: Treatment of excessive polycythemia of high altitude with respiratory stimulant drugs. Am Rev Respir Dis 117: 455-464, 1978.

11. Vargas M, Leon-Velarde F, Monge CC, Orozco E and Rey L: Enalapril in the treatment of chronic mountain sickness. Wilderness Environ Med 7: 193-194, 1996.

12. Plata R, Cornejo A, Arratia C, et al; Commission on Global Advancement of Nephrology (COMGAN), Research Subcommittee of the International Society of Nephrology: Angiotensin-converting-enzyme inhibition therapy in altitude polycythaemia: a prospective randomised trial. Lancet 359: 663-666, 2002.

13. Pelouch V, Kolár F, Ost'ádal B, Milerová M, Cihák R and Widimský J: Regression of chronic hypoxia-induced pulmonary hypertension, right ventricular hypertrophy, and fibrosis: effect of enalapril. Cardiovasc Drugs Ther 11: 177-185, 1997.

14. Richalet JP, Rivera-Ch M, Maignan M, et al: Acetazolamide for Monge's disease: efficiency and tolerance of 6-month treatment. Am J Respir Crit Care Med 177: 1370-1376, 2008.

15. Li P, Huang J, Tian HJ, Huang QY, Jiang CH and Gao YQ: Regulation of bone marrow hematopoietic stem cell is involved in high-altitude erythrocytosis. Exp Hematol 39: 37-46, 2011.

16. Gonzales GF, Gasco M, Tapia V and Gonzales-Castañeda C: High serum testosterone levels are associated with excessive erythrocytosis of chronic mountain sickness in men. Am J Physiol Endocrinol Metab 296: E1319-E1325, 2009.

17. Gonzales GF, Tapia V, Gasco M, Rubio J and Gonzales-Castañeda C: High serum zinc and serum testosterone levels were associated with excessive erythrocytosis in men at high altitudes. Endocrine 40: 472-480, 2011.

18. Bailey DM, Dehnert C, Luks AM, et al: High-altitude pulmonary hypertension is associated with a free radical-mediated reduction in pulmonary nitric oxide bioavailability. J Physiol 588: 4837-4847, 2010. 
19. Liggins J, Bluck LJ, Runswick S, Atkinson C, Coward WA and Bingham SA: Daidzein and genistein content of fruits and nuts. J Nutr Biochem 11: 326-331, 2000

20. Gencel VB, Benjamin MM, Bahou SN and Khalil RA: Vascular effects of phytoestrogens and alternative menopausal hormone therapy in cardiovascular disease. Mini Rev Med Chem 12: $149-174,2012$.

21. Hall WL, Formanuik NL, Harnpanich D, et al: A meal enriched with soy isoflavones increases nitric oxide-mediated vasodilation in healthy postmenopausal women. J Nutr 138: 1288-1292, 2008.

22. Kim H, Lee MJ, Kim JE, Park SD, Moon HI and Park WH: Genistein suppresses tumor necrosis factor-alpha-induced proliferation via the apoptotic signaling pathway in human aortic smooth muscle cells. J Agric Food Chem 58: 2015-2019, 2010.

23. Vera R, Galisteo M, Villar IC, et al: Soy isoflavones improve endothelial function in spontaneously hypertensive rats in an estrogen-independent manner: role of nitric-oxide synthase, superoxide, and cyclooxygenase metabolites. J Pharmacol Exp Ther 314: 1300-1309, 2005.

24. Li X, Gao Z, Liu F, Xiang D, Jiang C and Huang Q: The preventive effects of phytoestrogen on chronic hypoxic pulmonary hypertension in rats. Chinese Pharm Bull 20: 897-900, 2004 (In Chinese).

25. Li X, Gao Z, Liu F, Jiang C, Xiang D and Huang Q: The effects of genistein on pulmonary vascular structural remodeling induced by chronic hypoxia. Chinese Pharml Bull 20: 1257-1261, 2004 (In Chinese).
26. Li X, Gao Y, Liu F, et al: The regulative effects of genistein on vascular active factors in chronic hypoxic rats. Food Sci 20(10): 219-223, 2005 (In Chinese).

27. Allemann Y, Sartori C, Lepori M, et al: Echocardiographic and invasive measurements of pulmonary artery pressure correlate closely at high altitude. Am J Physiol Heart Circ Physiol 279: H2013-H2016, 2000.

28. Hamilton-Reeves JM, Vazquez G, Duval SJ, Phipps WR, Kurzer MS and Messina MJ: Clinical studies show no effects of soy protein or isoflavones on reproductive hormones in men: results of a meta-analysis. Fertil Steril 94: 997-1007, 2010.

29. Scherrer U, Vollenweider L, Delabays A, et al: Inhaled nitric oxide for high-altitude pulmonary edema. N Engl J Med 334: 624-629, 1996.

30. Hoit BD, Dalton ND, Erzurum SC, Laskowski D, Strohl KP and Beall CM: Nitric oxide and cardiopulmonary hemodynamics in Tibetan highlanders. J Appl Physiol 99: 1796-1801, 2005.

31. Ge RL, Mo VY, Januzzi JL, et al: B-type natriuretic peptide, vascular endothelial growth factor, endothelin-1, and nitric oxide synthase in chronic mountain sickness. Am J Physiol Heart Circ Physiol 300: H1427-1433, 2011. 